\title{
Additively manufactured metallic pentamode meta-materials
}

R. Hedayati, A. M. Leeflang, and A. A. Zadpoor

Citation: Appl. Phys. Lett. 110, 091905 (2017); doi: 10.1063/1.4977561

View online: http://dx.doi.org/10.1063/1.4977561

View Table of Contents: http://aip.scitation.org/toc/apl/110/9

Published by the American Institute of Physics

\section{Articles you may be interested in}

Complementary split-ring resonator antenna coupled quantum dot infrared photodetector

Appl. Phys. Lett. 110, 091106091106 (2017); 10.1063/1.4977427

Optofluidic Fano resonance photonic crystal refractometric sensors

Appl. Phys. Lett. 110, 091105091105 (2017); 10.1063/1.4977563

Shape formation of helical ribbons induced by material anisotropy

Appl. Phys. Lett. 110, 091901091901 (2017); 10.1063/1.4977090

Generating arbitrary ultrasound fields with tailored optoacoustic surface profiles

Appl. Phys. Lett. 110, 094102094102 (2017); 10.1063/1.4976942

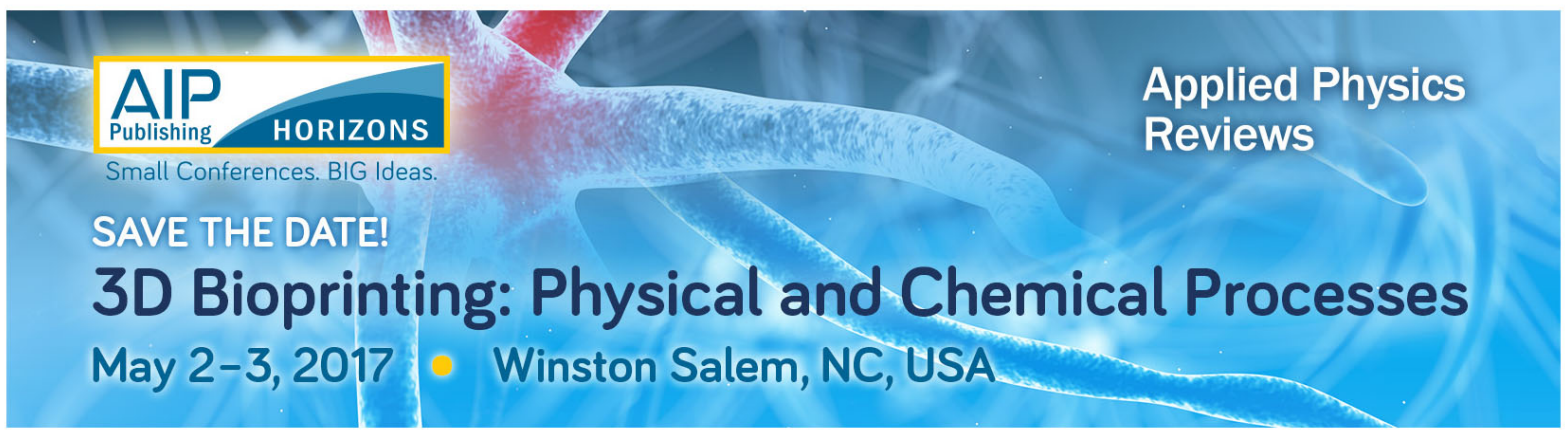




\title{
Additively manufactured metallic pentamode meta-materials
}

\author{
R. Hedayati, ${ }^{\text {a) }}$ A. M. Leeflang, and A. A. Zadpoor \\ Department of Biomechanical Engineering, Faculty of Mechanical, Maritime, and Materials Engineering, \\ Delft University of Technology (TU Delft), Mekelweg 2, 2628 CD Delft, The Netherlands
}

(Received 1 December 2016; accepted 14 February 2017; published online 28 February 2017)

\begin{abstract}
Mechanical metamaterials exhibit unusual mechanical properties that originate from their topological design. Pentamode metamaterials are particularly interesting because they could be designed to possess any thermodynamically admissible elasticity tensor. In this study, we additively manufacture the metallic pentamode metamaterials from a biocompatible and mechanically strong titanium alloy (Ti-6Al-4V) using an energy distribution method developed for the powder bed fusion techniques. The mechanical properties of the developed materials were a few orders of magnitude higher than those of the similar topologies fabricated previously from polymers. Moreover, the elastic modulus and yield stress of the presented pentamode metamaterials were decoupled from their relative density, meaning that the metallic meta-biomaterials with independently tailored elastic and mass transport (permeability) properties could be designed for tissue regeneration purposes. (C) 2017 Author(s). All article content, except where otherwise noted, is licensed under a Creative Commons Attribution (CC BY) license (http://creativecommons.org/licenses/by/4.0/).

[http://dx.doi.org/10.1063/1.4977561]
\end{abstract}

Mechanical metamaterials are rationally designed materials with unusual mechanical properties such as negative Poisson's ratio, negative elasticity, negative bulk modulus, and negative thermal expansion. ${ }^{1,2}$ The unusual mechanical properties of mechanical metamaterials are directly linked to (the topology of) their micro-architecture, which is why the process of rationally designing such materials can be often reduced to rationally designing the topology of their microarchitecture.

The so-called "extremal materials" were one of the first categories of mechanical metamaterials introduced by Milton and Cherkaev. ${ }^{3}$ They can be easily deformed in certain directions, while showing extremely high resistance against deformation in some other directions. The eigenvalues of the elasticity tensor approach either zero (i.e., extremely complaint) or infinity (i.e., extremely stiff) and determine the directions of extreme compliance and extreme stiffness. The extremal materials can be categorized according to the number of eigenvalues approaching zero as unimode, bimode, trimode, quadramode, or penta-mode. ${ }^{3}$ The pentamode mechanical materials can therefore be easily deformed in five of the six principal directions, meaning that their bulk modulus, $B$, is extremely large. An extremely large bulk modulus (i.e., incompressible) and the negligible resistance against shear have earned pentamode metamaterials the name "metafluids." 4,5

Among different classes of mechanical metamaterials, pentamodes are particularly important due to the fact that any thermodynamically admissible elasticity tensor could be realized through the consolidation of multiple pentamode topologies into one single lattice-based topology. ${ }^{3}$ As such, the pentamode metamaterials can be considered as a general platform for rational design of metamaterials with any desired elasticity tensor. In addition, the bulk modulus of pentamode metamaterials can be decoupled from their mass density, ${ }^{6}$ breaking down the Ashby-type power-law

a)Electronic addresses: r.hedayati@tudelft.nl and rezahedayati@gmail.com relationship observed between the elastic properties and mass density, which is known to hold for most materials. The decoupling of mass density and elastic properties offers additional flexibility in the rational design of mechanical metamaterials and highlights the practical importance of pentamodes as a general platform for the rational design of mechanical metamaterials.

Both the above-mentioned properties of pentamode metamaterials make them promising candidates for several practical applications including acoustic metamaterials ${ }^{6-8}$ as well as meta-biomaterials, ${ }^{9}$ which aim at improving tissue regeneration performance through the rational design of biomaterials with optimal combination of mechanical, mass transport, and biological properties. ${ }^{10,11}$

Even though very clear designs for pentamode metamaterials were proposed in 1995, they were not realized until 2012 (Ref. 12) when advanced additive manufacturing techniques enabled the production of such complex structures from polymeric materials. In the current study, we present additively manufactured pentamode metamaterials fabricated from a biocompatible titanium alloy (Ti-6Al-4V). In addition to being biocompatible and, thus, suitable for use in medical devices, the mechanical properties of this metallic alloy $\left(E=113 \mathrm{GPa}, \sigma_{y}=900 \mathrm{MPa}\right)$ are far superior to those of the polymeric materials $(E=3 \mathrm{GPa}$ (Ref. 6)) used before. A vector-based energy distribution technique has been proposed to enable the fabrication of the complex topology of pentamode metamaterials from metal powder. Pentamode metamaterials with different topological parameters are manufactured, characterized using mechanical testing, and compared with the predictions of our recently developed analytical model. Additive manufacturing of metallic pentamode meta-biomaterials paves the way for practical application of such materials as bone tissue engineering scaffolds and as parts of orthopedic implants.

The pentamode topology proposed by Milton and Cherkaev $^{3}$ is a lattice structure based on the diamond unit 
cell with struts that have a gradient in their thickness so as to form a double-cone shape (Figure $1(\mathrm{~d})$ ). We used a powder bed fusion technique, namely, selective laser melting (SLM) (Realizer SLM 125, Germany), for the fabrication of pentamode topology from the Ti-6Al-4V powder (medical grade, according to ASTM B348, grade 23). The metal powder was processed on top of a solid metal substrate under an inert (i.e., Argon) atmosphere. Metallic hierarchical structures with topological complexities similar to those of pentamode metamaterials have not been previously manufactured using the powder bed fusion techniques such as SLM because determining the laser processing parameters required for optimal energy distribution is a challenging task for such complex topologies. The density of the delivered energy should be high enough to ensure sufficiently overlapping melt pools with proper size to yield a fully solid matrix material, while avoiding overheating to prevent vaporization of the alloying elements and embrittlement of the resulting matrix material. Creating the required gradient in the diameter of the doublecone struts therefore requires a complex modification of the delivered energy density. An extensive preliminary study resulted in the development of a vector-based energy distribution strategy to create the required topologies. In this technique, an overlapping array of melt pools is created in the cross section of the struts (Figure 1(b)). However, the number of melting points and their offset was determined based on the criterion that no material point should be brought to the melting point for more than three times. This number was determined experimentally through a parametric study and ensured the material was not overheated and material embrittlement was avoided. For very thin cross-sections, only the central circle was used, and for larger cross-sections the
Low-energy density

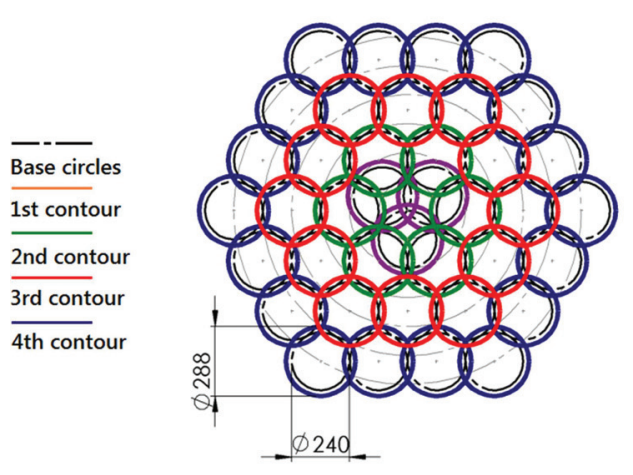

(a)

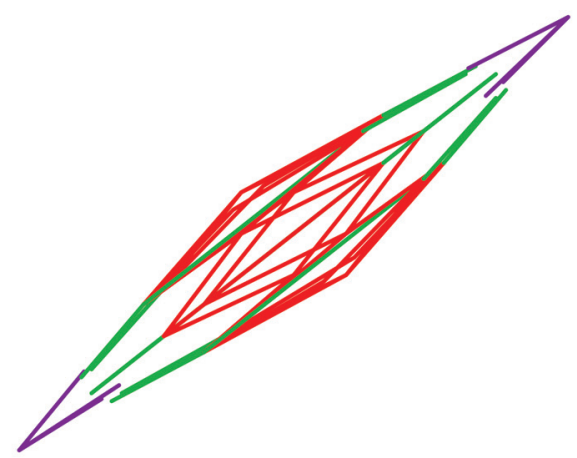

High-energy density
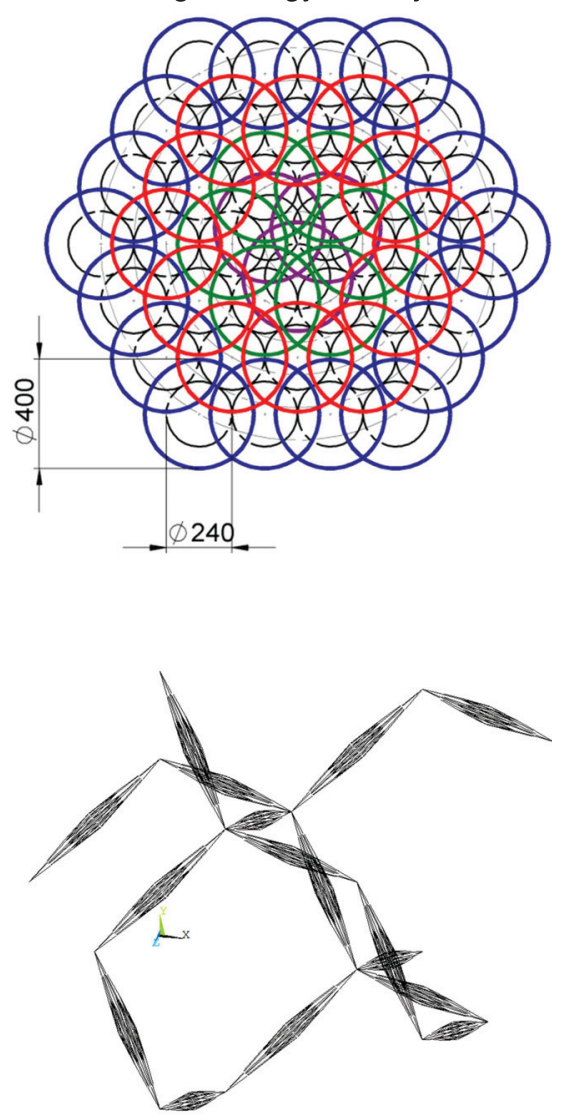

(c)
FIG. 1. (a) The diamond lattice structure used for constructing a pentamode metamaterial, (b) the evolution of weld points around the central circle for having different strut diameters (the dimensions are in micrometers), (c) and (d) the front and isometric views of the diamond unit cell, (e) the vector lines for creating weld points of the pentamode metamaterial, and (f) the vector lines in their volumetric view.

(b)

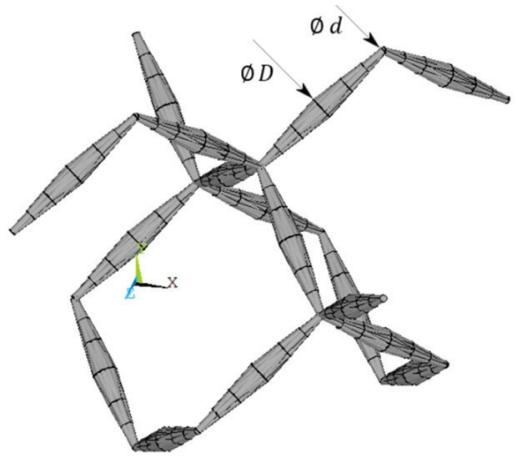

(d) 

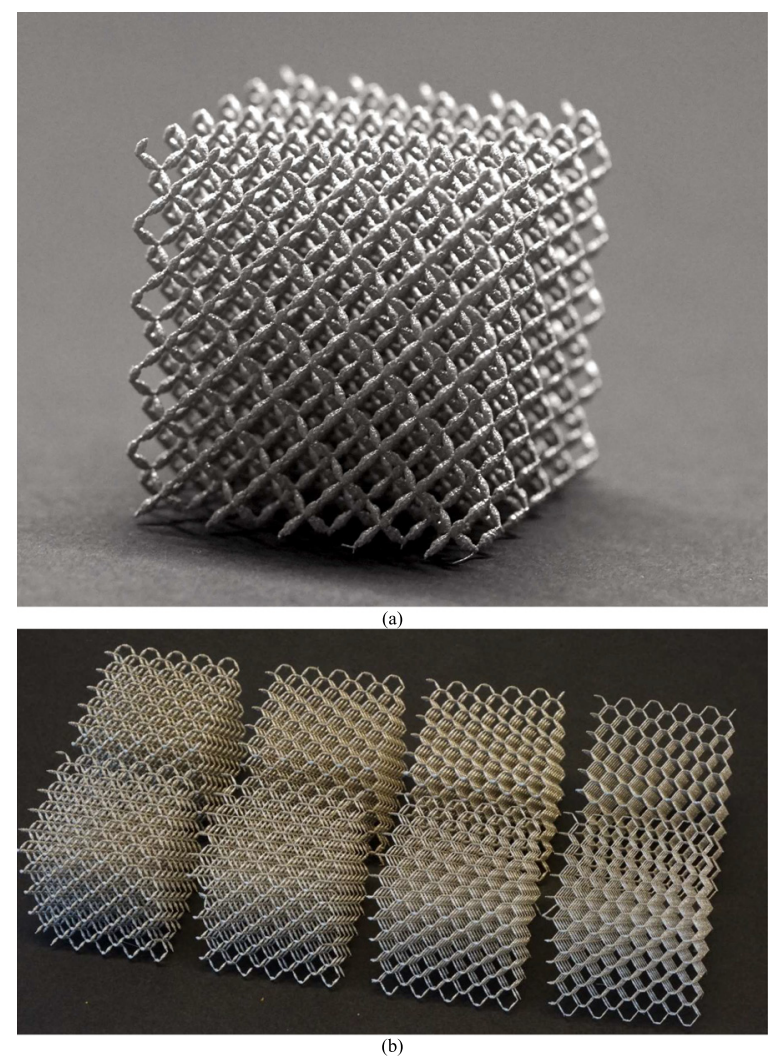

FIG. 2. (a) A pentamode metamaterial lattice structure from close view. (b) Pentamode metamaterials manufactured by different processing parameters and $\frac{D}{l}$ ratios. The top and bottom rows, respectively, belong to metamaterials manufactured by low and high energy density of the laser beam. The relative density decreases from left to right in each row.

welding points were evolved around the central circle to the total welding points of $7,19,39$, etc. As the diameter of the strut increased from the ends to the middle part, the central vectors were parallel to the axis of the strut and the additive external vectors made an angle with the strut axis (the external additive vectors can be seen in Figure 1(b)).

Pentamode metamaterials (Figure 2) with four different $D / l$ ratios and two different laser beam energy densities (low energy with an exposure time of $300 \mu$ s and laser current of $1200 \mathrm{~mA}$ and high energy with an exposure time of $550 \mu$ s and laser current of $1600 \mathrm{~mA}$ ) were manufactured. The different energy levels give rise to different melt pool sizes and, thus, different topological parameters (Table I, Figure 2). All specimens included 5 unit cells in each direction and were $4 \mathrm{~cm} \times 4 \mathrm{~cm} \times 4 \mathrm{~cm}$ (Figure 2). The nominal length of all the struts was $3.464 \mathrm{~mm}$. The manufactured samples were uniaxially compressed (displacement rate of $0.2 \mathrm{~mm} / \mathrm{min}$ ) using an INSTRON E10000 ElectroPulse dynamic mechanical test machine with a $10 \mathrm{kN}$ load cell. The obtained elastic modulus and yield stress values were determined in accordance to the ISO standard 13314:2011 and were normalized by dividing the noted values by those of the bulk material $\left(\mathrm{E}=113 \mathrm{GPa}\right.$ and $\left.\sigma_{y}=900 \mathrm{MPa}\right)$.

Weight measurements showed that the manufactured pentamodes had relative densities between $0.84 \%$ and $2.24 \%$ for low-energy density laser beam and between $1.54 \%$ and $4.24 \%$ for the high-energy density laser beam (Table I). As long as the energy level was kept constant, microscopic measurements (Figure 3) showed very close $d$ values for all the relative densities considered here (Table I). For example, in the specimens made using low-energy laser beam, the pentamode metamaterial with the lowest relative density structure had a $d$ value of $259 \pm 4 \mu \mathrm{m}$, while the pentamode with the highest relative density exhibited a $d$ value of $256 \pm 27 \mu \mathrm{m}$. The actual values of $\alpha\left(=\frac{d}{D}\right)$ were between 1.4 and 3.4 for the pentamodes manufactured with low energy laser beam and between 1.2 and 2.6 for the ones made with high energy laser beam (Table I), indicating a relative large span of $\alpha$ values. The stress-strain curves of the pentamode metamaterials made with the same laser energy density were very close (Figure 4(a)). The elastic modulus and yield stress of the pentamode metamaterials were several (i.e., 4-6) orders of magnitude smaller than those of the matrix material from which they were made (Figures 4(b) and 4(c)). The elastic modulus (Figure 4(b)) and yield stress (Figure 4(c)) were similar as long as the same energy level was used and regardless of $D$ values.

We compared the elastic modulus, $E$, and yield stress, $\sigma_{y}$, measured for the additively manufactured pentamode metamaterials with the analytical predictions obtained for a lattice structure based on the diamond unit cell ${ }^{13,14}$

$$
\begin{gathered}
\frac{E}{E_{s}}=\frac{\sqrt{6} \pi\left(\frac{3}{4}\right)^{2}\left(\frac{r}{l}\right)^{4}}{1+\frac{3}{2}\left(\frac{r}{l}\right)^{2}}, \\
\frac{\sigma_{y}}{\sigma_{y s}}=\frac{9 \pi}{4 \sqrt{6}}\left(\frac{r}{l}\right)^{3},
\end{gathered}
$$

\begin{tabular}{|c|c|c|c|c|c|c|c|}
\hline Name & $d$, design $(\mu \mathrm{m})$ & $d$, actual $(\mu \mathrm{m})$ & $D$, design $(\mu \mathrm{m})$ & $D$, actual $(\mu \mathrm{m})$ & $\alpha$, design & $\alpha$, actual & Relative density (\%) \\
\hline \multicolumn{8}{|c|}{ Low-energy density } \\
\hline Density 1 & 288 & $259 \pm 4$ & 408 & $369 \pm 22$ & 1.42 & 1.42 & 0.84 \\
\hline Density 2 & 288 & $256 \pm 4$ & 648 & $505 \pm 4$ & 2.25 & 1.97 & $1.58 \pm 0.01$ \\
\hline Density 3 & 288 & $246 \pm 8$ & 888 & $682 \pm 18$ & 3.08 & 2.77 & $1.94 \pm 0.00$ \\
\hline Density 4 & 288 & $256 \pm 27$ & 1128 & $882 \pm 18$ & 3.92 & 3.44 & $2.24 \pm 0.04$ \\
\hline \multicolumn{8}{|c|}{ High-energy density } \\
\hline Density 1 & 400 & $372 \pm 25$ & 568 & $433 \pm 29$ & 1.42 & 1.16 & $1.54 \pm 0.02$ \\
\hline Density 2 & 400 & $321 \pm 17$ & 808 & $654 \pm 24$ & 2.02 & 2.03 & $2.96 \pm 0.15$ \\
\hline Density 3 & 400 & $354 \pm 15$ & 1048 & $782 \pm 9$ & 2.62 & 2.21 & $3.31 \pm 0.16$ \\
\hline Density 4 & 400 & $341 \pm 12$ & 1288 & $902 \pm 23$ & 3.22 & 2.64 & $4.24 \pm 0.02$ \\
\hline
\end{tabular}

TABLE I. Geometrical properties and measured mechanical properties of pentamode metamaterials made by low-energy density laser beam. 

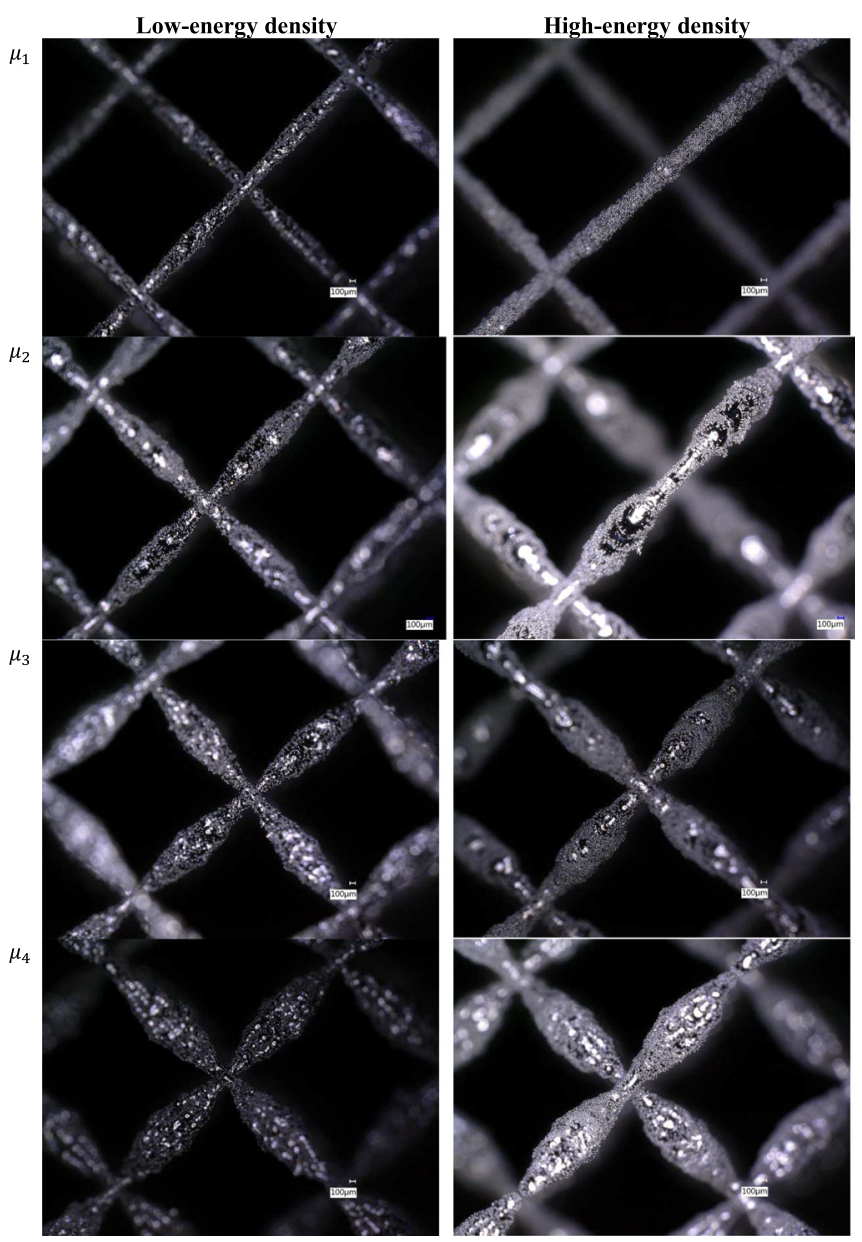

FIG. 3. The microscopic views of struts in the SLM made pentamode metamaterials with different designs and laser beam energy density.

where the subscript $s$ refers to the matrix (solid) matrix from which the lattice material is made. In Equations (1) and (2), we used the actual $r=d / 2$ values to calculate the normalized elastic modulus and yield stress of the pentamode metamaterials. The normalized elastic modulus calculated using Equations (1) and (2) was generally very close to the experimentally determined values (Figure 4(b); compare the experimental data points with the line showing the analytical solution). The differences between the experimentally determined values of the normalized yield stress and the corresponding values predicted by the analytical solutions were somewhat higher than those observed for the normalized elastic modulus (Figure 4(c)). In almost all cases, the analytical model predicts higher values of normalized yield stress, as compared to what was observed experimentally (Figure $4(c)$ ). These relatively lower values of mechanical properties are in line with the previous observations for the same additive manufacturing technique and can be attributed to the manufacturing imperfections (the presence of irregularity in the external surface of the struts as well as the micro-pores created inside the struts during melting of the powders). ${ }^{15-17}$ In particular, similar to the results in this study, the difference between the analytical and experimental results was larger for the yield stress. ${ }^{15-19}$

The above-mentioned results clearly show that the mechanical properties of the metallic pentamode metamaterials fabricated in the current study are not dependent on the
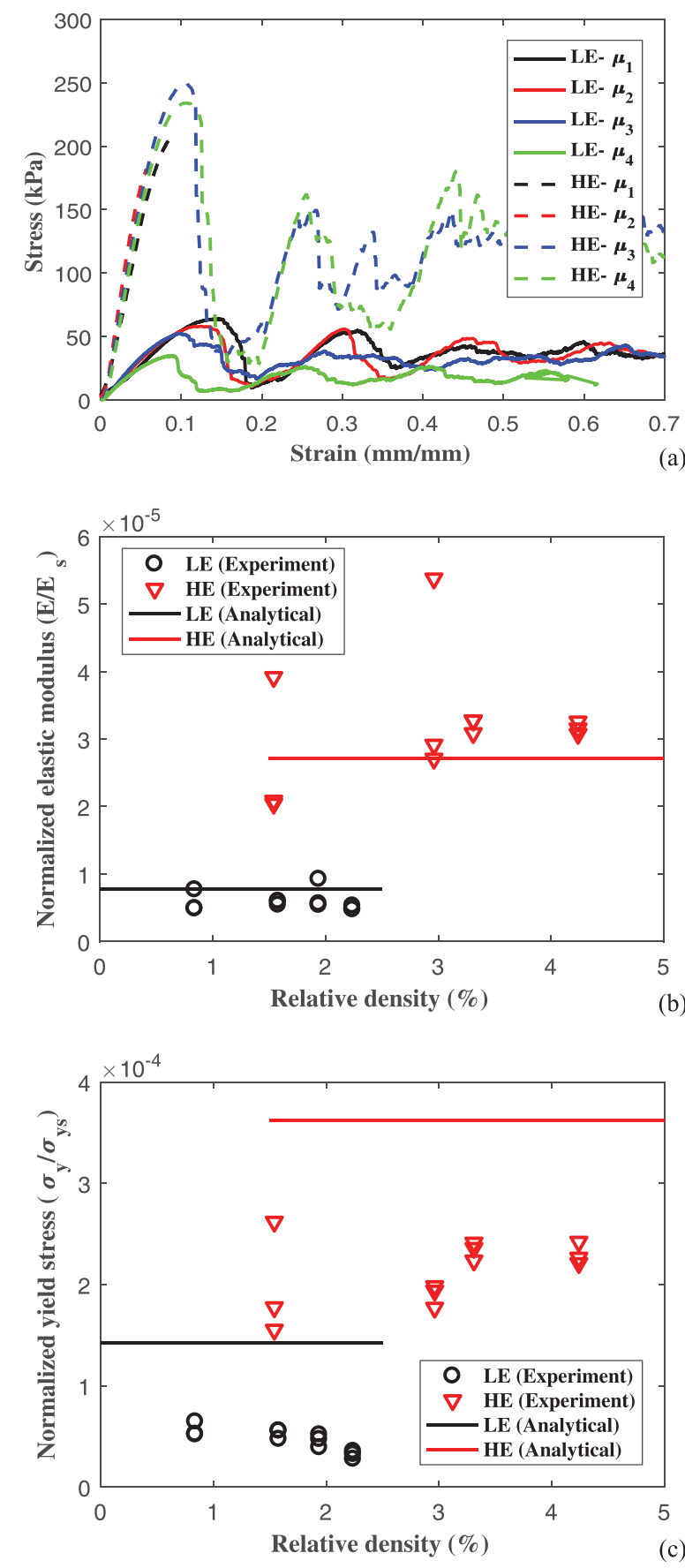

FIG. 4. Comparison of (a) stress-stress curves, (b) elastic modulus vs. relative density, and (c) yield stress vs. relative density for SLM made pentamode metamaterials with different designs and laser beam energy density (LE is the low-energy density and HE is the high-energy density). The stressstrain curve belonging to each specimen type is plotted up to the point, where the stress-strain curve of all the three specimens was available (i.e., up to the strain at which all the three specimens were not broken).

relative density and are merely related to the smaller diameter, $d$. This has important implications for the application of metallic pentamode metamaterials in biomedical applications because the larger diameter, $D$, and the relative density are, nevertheless, related to the mass transport properties of the pentamode metamaterials, such as its permeability. This break-down of the Ashby-type relationship between mass density and elastic properties therefore enables us to decouple the mechanical properties of meta-biomaterials from their 
mass transport properties. Given the fact that mass transport properties play an important role in bone tissue regeneration performance of biomaterials particularly in the initial phases of tissue regeneration when blood vessels have not yet been formed, ${ }^{10}$ the ability to decouple elastic properties and mass transport properties allows for optimal design of metabiomaterials so as to simultaneously achieve sufficient mechanical stimulus for bone tissue regeneration and sufficient mass transport for cell oxygenation and nutrition.

The absolute values of the elastic modulus of the metallic pentamode metamaterials manufactured here were in the range between 506.9 and $942.5 \mathrm{kPa}$ for the low energy laser beam and between 3.642 and $7.603 \mathrm{MPa}$ for the high energy laser beam. In comparison, the absolute elastic moduli of the polymeric pentamode structures manufactured by dip-in direct-laser-writing (DLW) optical lithography were in the range between 0.105 and $428 \mathrm{kPa}^{12}$ Given the much higher elastic modulus of the bulk metallic material used in the current study, i.e., $110 \mathrm{GPa}$, as compared to the elastic modulus of the polymeric material used in the previous studies, i.e., $3 \mathrm{GPa}$, the elastic modulus of the resulting pentamode metamaterials is much higher than what could be previously achieved. Much higher stiffness values are critical for the practical application of pentamode metamaterials in general and for their biomedical application in particular. For example, in order to generate pentamode metamaterials that could be used as bone substitutes and, thus, have mechanical properties similar to those of bone, much higher relative densities are required. In a metallic pentamode metamaterial made from Ti-6Al-4V, relative elastic moduli between 0.01 and 0.1 are required to achieve elastic moduli between $1 \mathrm{GPa}$ (corresponding to cancellous bone) and $10 \mathrm{GPa}$ (corresponding to cortical bone). To exhibit such high values of elastic modulus, the ratio of the smaller diameter to the strut length $(r / l)$ of the pentamode metamaterial should be between 0.22 and 0.41 , as calculated using the inverse of Equation (1). In comparison, that ratio was between 0.04 and 0.05 for the pentamode metamaterials manufactured in the current study. It is therefore suggested that the future studies manufacture metallic pentamode metamaterials with larger minimum diameters, so as to achieve elastic moduli comparable to those of human bone. Since the relative density of the pentamode metamaterials has been very small in all the previous studies on the mechanical properties of pentamode metamaterials, ${ }^{6,12,20,21}$ it is also suggested to investigate whether or not the statement "the stiffness of penta-mode metamaterials is only dependent on the smaller diameter size of the struts" ${ }^{12}$ holds true also for pentamode metamaterials with much higher values of relative density.

In summary, the metallic pentamode metamaterials were additively manufactured from a biocompatible titanium alloy using a vector-based energy distribution strategy developed here for selective laser melting, i.e., the powder bed fusion additive manufacturing technique. The compressive mechanical properties of the metallic pentamode metamaterials were found to be several times higher than those reported for polymeric pentamode metamaterials. Moreover, the mechanical properties of the developed metallic pentamode metamaterials were independent from their relative density, meaning that the mechanical and mass transport properties of metallic pentamode metamaterials can be decoupled from each other. This decoupling of elastic modulus and permeability has important implications for biomedical applications of such materials.

${ }^{1}$ J. H. Lee, J. P. Singer, and E. L. Thomas, "Micro-/nanostructured mechanical metamaterials," Adv. Mater. 24, 4782-4810 (2012).

${ }^{2}$ A. A. Zadpoor, "Mechanical meta-materials," Mater. Horiz. 3, 371-381 (2016).

${ }^{3}$ G. W. Milton and A. V. Cherkaev, "Which elasticity tensors are realizable?," J. Eng. Mater. Technol. 117, 483-493 (1995).

${ }^{4}$ C. N. Layman, C. J. Naify, T. P. Martin, D. C. Calvo, and G. J. Orris, "Highly anisotropic elements for acoustic pentamode applications," Phys. Rev. Lett. 111, 024302 (2013).

${ }^{5}$ A. N. Norris, "Acoustic metafluids," J. Acoust. Soc. Am. 125, 839-849 (2009).

${ }^{6}$ M. Kadic, T. Bückmann, R. Schittny, P. Gumbsch, and M. Wegener, "Pentamode metamaterials with independently tailored bulk modulus and mass density," Phys. Rev. Appl. 2, 054007 (2014).

${ }^{7}$ M. Kadic, T. Bückmann, R. Schittny, and M. Wegener, "On anisotropic versions of three-dimensional pentamode metamaterials," New J. Phys. 15, 023029 (2013).

${ }^{8}$ A. Martin, M. Kadic, R. Schittny, T. Bückmann, and M. Wegener, "Phonon band structures of three-dimensional pentamode metamaterials," Phys. Rev. B 86, 155116 (2012).

${ }^{9}$ S. Amin Yavari, S. Ahmadi, R. Wauthle, B. Pouran, J. Schrooten, H. Weinans, and A. Zadpoor, "Relationship between unit cell type and porosity and the fatigue behavior of selective laser melted meta-biomaterials," J. Mech. Behav. Biomed. Mater. 43, 91-100 (2015).

${ }^{10}$ A. A. Zadpoor, "Bone tissue regeneration: The role of scaffold geometry," Biomater. Sci. 3, 231-245 (2015).

${ }^{11}$ R. Hedayati, M. Sadighi, M. Mohammadi-Aghdam, and A. Zadpoor, "Analytical relationships for the mechanical properties of additively manufactured porous biomaterials based on octahedral unit cells," Appl. Math. Modell. 46, 408 (2017).

${ }^{12}$ M. Kadic, T. Bückmann, N. Stenger, M. Thiel, and M. Wegener, "On the practicability of pentamode mechanical metamaterials," Appl. Phys. Lett. 100, 191901 (2012).

${ }^{13}$ S. Ahmadi, G. Campoli, S. Amin Yavari, B. Sajadi, R. Wauthlé, J. Schrooten, H. Weinans, and A. A. Zadpoor, "Mechanical behavior of regular open-cell porous biomaterials made of diamond lattice unit cells," J. Mech. Behav. Biomed. Mater. 34, 106-115 (2014).

${ }^{14}$ A. A. Zadpoor and R. Hedayati, "Analytical relationships for prediction of the mechanical properties of additively manufactured porous biomaterials," J. Biomed. Mater. Res. Part A 104, 3164-3174 (2016).

${ }^{15}$ R. Hedayati, M. Sadighi, M. Mohammadi-Aghdam, and A. Zadpoor, "Mechanical properties of regular porous biomaterials made from truncated cube repeating unit cells: Analytical solutions and computational models," Mater. Sci. Eng. C 60, 163-183 (2016).

${ }^{16}$ R. Hedayati, M. Sadighi, M. Mohammadi-Aghdam, and A. Zadpoor, "Mechanical behavior of additively manufactured porous biomaterials made from truncated cuboctahedron unit cells," Int. J. Mech. Sci. 106, 19-38 (2016).

${ }^{17}$ R. Hedayati, M. Sadighi, M. Mohammadi-Aghdam, and A. Zadpoor, "Mechanics of additively manufactured porous biomaterials based on the rhombicuboctahedron unit cell," J. Mech. Behav. Biomed. Mater. 53, 272-294 (2016).

${ }^{18}$ S. Van Bael, G. Kerckhofs, M. Moesen, G. Pyka, J. Schrooten, and J.-P. Kruth, "Micro-CT-based improvement of geometrical and mechanical controllability of selective laser melted Ti6Al4V porous structures," Mater. Sci. Eng. A 528, 7423-7431 (2011).

${ }^{19}$ C. Yan, L. Hao, A. Hussein, P. Young, and D. Raymont, "Advanced lightweight $316 \mathrm{~L}$ stainless steel cellular lattice structures fabricated via selective laser melting," Mater. Des. 55, 533-541 (2014).

${ }^{20}$ G. F. Méjica and A. D. Lantada, "Comparative study of potential pentamodal metamaterials inspired by Bravais lattices," Smart Mater. Struct. 22, 115013 (2013).

${ }^{21}$ R. Schittny, T. Bückmann, M. Kadic, and M. Wegener, "Elastic measurements on macroscopic three-dimensional pentamode metamaterials," Appl. Phys. Lett. 103, 231905 (2013). 